\title{
Observation of strong magnetoelastic coupling in a first-order phase transition of $\mathrm{CrOCl}$
}

\author{
Joachim Angelkort, Alexander Wölfel, Andreas Schönleber, and Sander van Smaalen* \\ Laboratory of Crystallography, University of Bayreuth, 95440 Bayreuth, Germany \\ Reinhard K. Kremer \\ MPI for Solid State Research, Heisenbergstrasse 1, 70569 Stuttgart, Germany
}

(Received 2 July 2009; revised manuscript received 11 September 2009; published 20 October 2009)

\begin{abstract}
The phase transition of $\mathrm{CrOCl}$ toward a state of antiferromagnetic order below $T_{N}=13.5 \mathrm{~K}$ has been identified as a first-order phase transition. The transition is accompanied by a lattice and structural distortion toward a twofold, 2b nuclear superstructure with a-axis unique monoclinic symmetry, as evidenced by temperature-dependent $\mathrm{x}$-ray diffraction experiments. Magnetic-susceptibility and magnetization measurements indicate a transition with strong magnetoelastic coupling to a uniaxial antiferromagnet with ordered moments along c. A second transition is discovered at $T_{c} \approx 27.2 \mathrm{~K}$ that is presumably of purely magnetic origin and might indicate the formation of an incommensurate magnetic superstructure. The different behaviors of $\mathrm{TiOCl}, \mathrm{VOCl}$, and $\mathrm{CrOCl}$ are the result of the different symmetries of the filled $3 d$ orbitals, which lead to different exchange interactions on the $M O$ double layers of these isostructural compounds.
\end{abstract}

DOI: 10.1103/PhysRevB.80.144416

PACS number(s): $61.50 . \mathrm{Ks}, 61.66 . \mathrm{Fn}, 75.30 . \mathrm{Kz}$

\section{INTRODUCTION}

TiOCl has recently attracted attention because of the presence of quasi-one-dimensional $S=1 / 2$ magnetic chains of $\mathrm{Ti}$ atoms, which are responsible for the development of a spinPeierls state below $T_{c 1}=67 \mathrm{~K} .^{1,2}$ The low-dimensional character of the magnetic interactions in $\mathrm{TiOCl}$ is the result of the presence of the single $d$ electron of $\mathrm{Ti}^{3+}$ in the $3 d_{x^{2}-y^{2}}$ orbital, which then gives rise to a large direct exchange along the chains of metal atoms but allows only weak magnetic exchange between the chains. ${ }^{3}$ Several compounds $\mathrm{MOCl}$ (where $M$ is a $3 d$ transition metal) are known to be isostructural to $\mathrm{TiOCl}$ at room temperature. ${ }^{4-7}$ They differ from each other in the number of $d$ electrons on the $M^{3+}$ atom. Different phase diagrams may be expected and have indeed been observed ${ }^{8-10}$ Compounds $\mathrm{MOCl}$ are thus of interest because variation in the element $M$ allows the magnetic interactions to be varied on otherwise equal lattices.

Isostructural compounds $M \mathrm{O} X(M=\mathrm{Ti}, \mathrm{V}, \mathrm{Cr}, \mathrm{Fe} ; X=\mathrm{Cl}$, $\mathrm{Br})$ crystallize in the $\mathrm{FeOCl}$ structure type with space group Pmmn and lattice parameters $a=3.8638(2), b=3.1793(1)$, and $c=7.7157(3) \AA$ for $\mathrm{CrOCl}$ at room temperature. ${ }^{4}$ The crystal structures consist of $M O$ double layers sandwiched between layers of halogen atoms which stack along the lattice direction c (Fig. 1). Another view of the structure is that of ribbons $M \mathrm{O} X$ perpendicular to $\mathbf{a}$. Within each ribbon a chain of metal atoms runs along $\mathbf{b}$. The short $M-M$ distances $(d=3.179 \AA)$ along these chains allow direct exchange interactions between electrons on neighboring atoms. ${ }^{3}$ Direct exchange between $\mathrm{Ti}$ atoms has been discussed as the reason for the unusual phase diagrams of $\mathrm{TiOCl}$ and $\mathrm{TiOBr}$. However, the shortest $M-M$ bond $(d=3.006 \AA)$ is between ribbons, i.e., between $\mathrm{Cr} 1$ and $\mathrm{Cr} 3$ in Fig. 1. Depending on the symmetry of the filled $d$ orbitals, direct exchange between ribbons as well as superexchange between ribbons and superexchange along a must be considered.

In $\mathrm{TiOCl}$ and $\mathrm{TiOBr}$ a spin-Peierls transition has been observed inducing a lowering of the orthorhombic to mono- clinic symmetry. The low-temperature spin-Peierls phases in $\mathrm{TiOCl}$ and $\mathrm{TiOBr}$ have a-axis unique monoclinic symmetry. ${ }^{2}$ In addition both compounds exhibit an intermediate phase between $T_{c 1}$ and $T_{c 2}$-with $T_{c 2}=90 \mathrm{~K}$ for $\mathrm{TiOCl}$ - which is incommensurately modulated with $\mathbf{c}$-axis unique monoclinic symmetry. ${ }^{11-13}$ Recently it was found that VOCl displays an c-axis unique monoclinic lattice distortion below $T_{N}$ $=80.3 \mathrm{~K}$, which supports antiferromagnetic (AFM) order with a twofold magnetic superstructure. ${ }^{14,15}$ The formation of this phase can be explained by the filling of two $3 d$ orbitals by the two $d$ electrons of $\mathrm{V}^{3+}$.

$\mathrm{CrOCl}$ was reported to develop AFM order at low temperatures with a fourfold magnetic superstructure. ${ }^{8}$ Here we report the temperature dependence of the anisotropic magnetic susceptibility, which indicates that ordered magnetic moments are parallel to c. X-ray diffraction experiments reveal that the magnetic transition to the AFM state is accompanied by an a-axis unique monoclinic lattice distortion. The transition to the magnetically ordered state is a first-order transition and it is accompanied by a structural distortion toward a twofold superstructure. By combining temperaturedependent and field-dependent magnetization and specificheat measurements we arrive at a proposal of the $(H, T)$

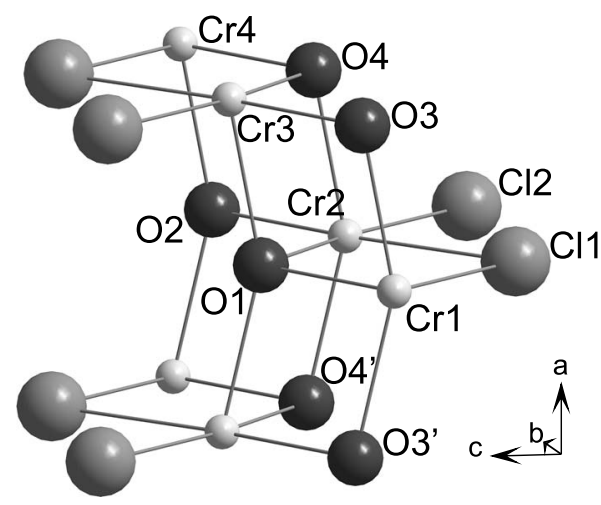

FIG. 1. Crystal structure of CrOCl. 
phase diagram of $\mathrm{CrOCl}$. The differences in phase diagrams of different compounds $\mathrm{MOCl}$ are discussed in view of the different numbers of $d$ electrons of the metal atoms.

\section{EXPERIMENT}

Single crystals of $\mathrm{CrOCl}$ were grown in evacuated quartzglass ampoules by gas transport according to published procedures. ${ }^{4,16}$ Stoichiometric amounts of $\mathrm{Cr}_{2} \mathrm{O}_{3}$ (Alpha, 99.997\% purity) and $\mathrm{CrCl}_{3}$ (Alpha, 99.9\%) were mixed with $\mathrm{HgCl}_{2}$ as transport agent and placed in a sealed and evacuated quartz-glass ampoule. The ampoule was heated for five days in a temperature gradient of $1223 \mathrm{~K}$ (educt side) to 1123 $\mathrm{K}$ (product side). $\mathrm{CrOCl}$ formed at the product side of the ampoule as greenish platelike crystals of up to several millimeter in length. The sample also contained smaller amounts of a dark green powder $\left(\mathrm{Cr}_{2} \mathrm{O}_{3}\right)$ on the educt side and $\mathrm{CrCl}_{3}$ on the product side.

Magnetic susceptibilities at constant field and varying temperature and magnetizations at constant temperature and varying field of a single crystal of $0.45 \mathrm{mg}$ were measured in a Quantum Design Magnetic Properties Measurement System (MPMS) squid magnetometer with the magnetic field oriented either parallel or perpendicular to $\mathbf{c}$. Heat capacities of the same crystal were determined with the magnetic field oriented parallel to $\mathbf{c}$ in a Quantum Design Physical Properties Measurement System (PPMS) employing the relaxation method.

A crystal of dimensions $0.15 \times 0.05 \times 0.005 \mathrm{~mm}^{3}$ was selected for $\mathrm{x}$-ray diffraction experiments using synchrotron radiation of wavelength $0.71000 \AA$. The crystal was glued to a carbon fiber that was attached to the cold finger of a closedcycle helium cryostat mounted on the Huber four-circle diffractometer at beamline D3 of Hasylab at DESY, Hamburg, Germany, equipped with a scintillation detector. Diffraction was measured at selected temperatures between $8 \mathrm{~K}$ and room temperature.

\section{RESULTS}

\section{A. Magnetic susceptibility and magnetization}

Above $\sim 175 \mathrm{~K}$ the magnetic susceptibilities follow a Curie-Weiss law according to (Fig. 2)

$$
\chi_{\text {mol }}=\frac{C}{T-\Theta}+\chi_{\text {dia }}
$$

with a Curie constant $C=1.825(9) \mathrm{cm}^{3} \mathrm{~K} / \mathrm{mol}$, equivalent to an effective magnetic moment $\mu_{e f f}=3.82(1) \mu_{B}$, in good agreement with previous measurements. ${ }^{4}$ When fitting Eq. (1) to the data, we fixed the diamagnetic contribution to $\chi_{\text {dia }}=-49 \times 10^{-6} \mathrm{~cm}^{3} / \mathrm{mol}$, corresponding to the sum of the diamagnetic increments of the constituting elements in their respective oxidation states according to Selwood, ${ }^{17} \mathrm{Cr}^{3+}: 11$ $\times 10^{-6} \mathrm{~cm}^{3} / \mathrm{mol} ; \quad \mathrm{O}^{2-}: 12 \times 10^{-6} \mathrm{~cm}^{3} / \mathrm{mol} ; \quad \mathrm{Cl}^{-}: 26 \times 10^{-6}$ $\mathrm{cm}^{3} / \mathrm{mol}$. The effective moment is in good agreement with the value expected for a $3 d^{3}$ electronic configuration with spin-only $S=3 / 2$ state of the $\mathrm{Cr}^{3+}$ ion and a $g$ factor of $g$ $\approx 1.97$ somewhat reduced from the free-electron $g$ factor due to spin-orbit coupling effects. ${ }^{18}$ The Curie-Weiss temperature

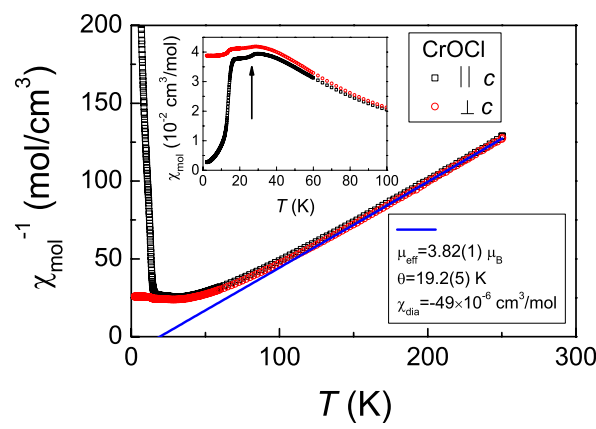

FIG. 2. (Color online) Magnetic susceptibility of $\mathrm{CrOCl}$ measured in a magnetic field of $0.1 \mathrm{~T}$ parallel and perpendicular to $\mathbf{c}$. The (blue) solid line represents a fit of the Curie-Weiss law [Eq. (1)] to the data at temperatures $T>175 \mathrm{~K}$ (see text). The small but finite value of $\chi_{\|}$for $T \rightarrow 0$ is attributed to a slight misalignment of the crystal. The arrow in the inset indicates a weak anomaly near $T_{c} \approx 27.2 \mathrm{~K}$.

is positive and amounts to $\Theta=19.2(5) \mathrm{K}$, indicating predominant ferromagnetic exchange interaction.

Below about $80 \mathrm{~K}$ the susceptibilities measured with fields parallel and perpendicular to the $\mathbf{c}$ axis increasingly deviate from each other. A broad maximum appears at $\sim 30 \mathrm{~K}$ for both directions of the field. Below $\sim 14 \mathrm{~K} \chi_{\|}$ drops almost to zero while $\chi_{\perp}$ levels off at a value of 3.88 $\times 10^{-2} \mathrm{~cm}^{3} / \mathrm{mol}$ indicating long-range AFM ordering in agreement with previous findings. ${ }^{8}$ The splitting of parallel and perpendicular susceptibilities is consistent with the notion of an easy-axis antiferromagnet with the easy axis along or close to the crystallographic c axis.

Onset of AFM long-range ordering below $\sim 14 \mathrm{~K}$ is marked by an anomaly at $13.9(1) \mathrm{K}$ in the specific heat $\left(C_{p}\right)$ measured on the same crystal as used for the determination of the magnetic susceptibilities. An additional, split anomaly of about the same magnitude is observed at 26.7 and $27.8 \mathrm{~K}$ (Fig. 3).

Magnetization experiments with the magnetic field applied along the easy axis revealed a spin-flop transition at a flop field $H_{S F}=3.2(1) \mathrm{T}$ (Fig. 4). A hysteresis is observed between the magnetization measured with increasing and decreasing fields. The derivative $d M / d H$ clearly reveals the splitting of the transition measured with increasing and de-

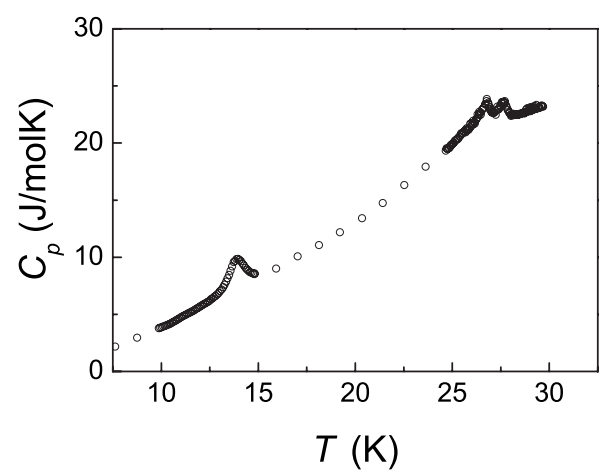

FIG. 3. Temperature dependence of the specific heat $C_{p}$ of $\mathrm{CrOCl}$. Anomalies are clearly revealed at 13.9(1) $\mathrm{K}$ and at 26.7 and $27.8 \mathrm{~K}$. 


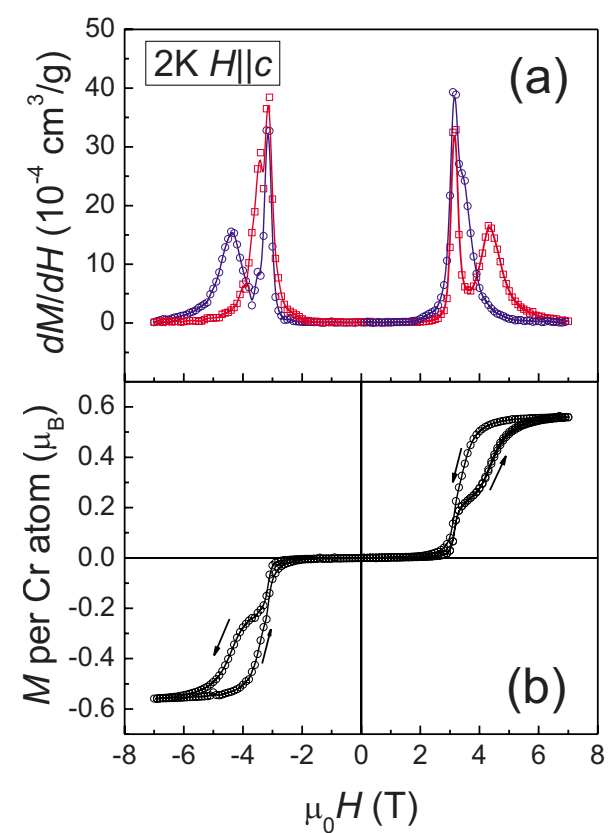

FIG. 4. (Color online) Magnetization $M$ and its derivative with respect to the magnetic field, $d M / d H$, measured on $\mathrm{CrOCl}$ at $T$ $=2 \mathrm{~K}$ with the magnetic field applied along the $\mathbf{c}$ axis. The hysteresis in the magnetization branches with data collected by increasing and decreasing field (as indicated by the arrows) is clearly visible and symmetric if the field is reversed. (a) solid (red) lines and symbols $\square$ mark values obtained on increasing the external field while (blue) solid lines and symbols $\bigcirc$ mark values obtained with decreasing external field.

creasing fields to be on the order of $1.3 \mathrm{~T}$ [Fig. 4(a)]. Combining the information obtained from magnetization and specific-heat experiments enables us to construct the $(H, T)$ phase diagram for the magnetic phases of $\mathrm{CrOCl}$ as shown in Fig. 5.

\section{B. X-ray diffraction}

Preliminary x-ray diffraction experiments confirmed the $\mathrm{FeOCl}$ structure type of $\mathrm{CrOCl}$. The first series of synchrotron experiments was carried out at selected temperatures in a cooling cycle from 270 to $9 \mathrm{~K}$. At each temperature so-

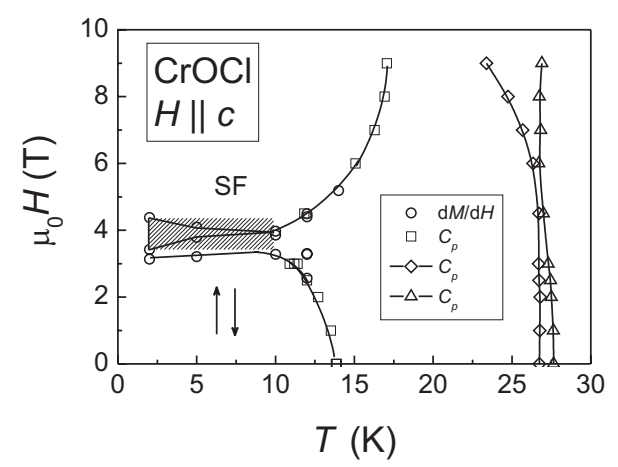

FIG. 5. Phase diagram of $\mathrm{CrOCl}$ with the magnetic field applied along the crystallographic $\mathbf{c}$ axis. The spin-flop phase is indicated by SF. The shaded area indicates the hysteresis of the spin-flop transition.
Reflection 0 -2 5
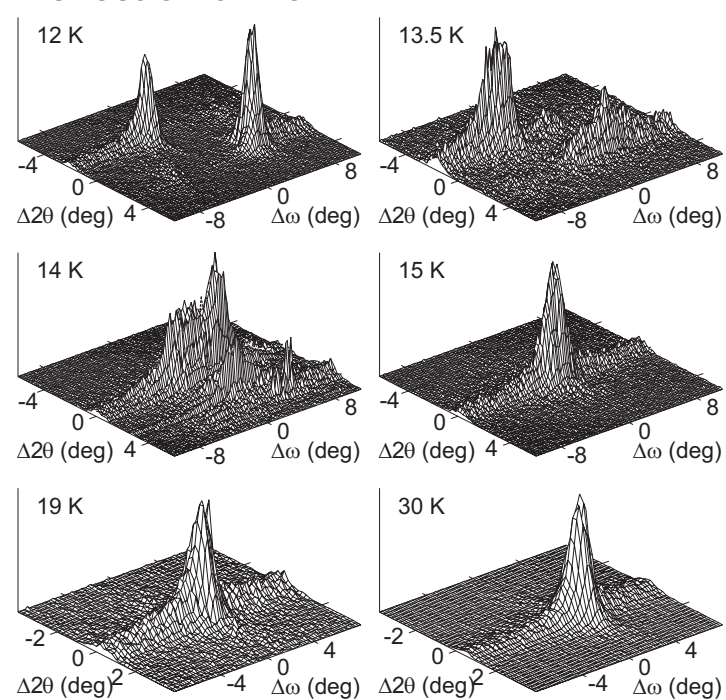

Reflection 204

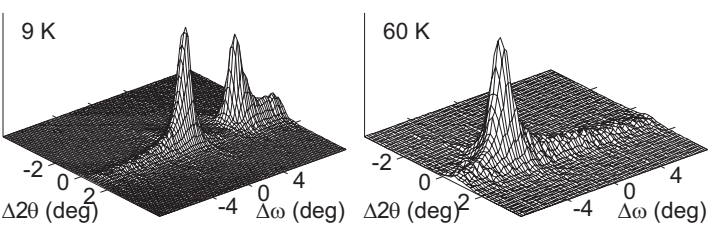

Reflection 2 -2 0
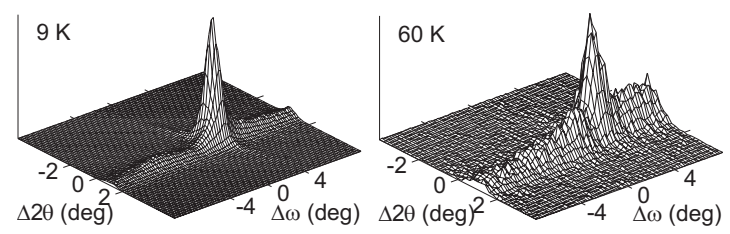

FIG. 6. Diffracted intensity as a function of the scattering angle $2 \theta$ and the crystal orientation $\omega$ for several reflections at selected temperatures. $\Delta 2 \theta$ and $\Delta \omega$ indicate the deviation from the center of the scan in units of $0.01^{\circ}$.

called $\omega$-2 $\theta$ maps were measured for the reflections $(0 \overline{2} 5),(2$ $04)$, and $(2 \overline{2} 0)$. To this end, detector slits of $6 \times 0.02 \mathrm{~mm}^{2}$ were set, which correspond to an acceptance angle in the direction of $2 \theta$ of $0.0031^{\circ}$. $\omega$ scans (rotation of the crystal) were carried out for a series of $2 \theta$ values, with step sizes of $0.002^{\circ}$ in both $\omega$ and $2 \theta$. The resulting plot shows the diffracted intensity as a function of the orientation of the crystal and as a function of the scattering angle. Within the orthorhombic lattice all reflections are expected as single peaks, as is indeed observed down to $T=15 \mathrm{~K}$ (Fig. 6). Some scans show a slight broadening of the peaks in the direction of $\omega$, which indicates a less than optimal crystal quality, most likely resulting from anisotropic stress induced by different thermal contractions of the sample and the glue used to attach the sample.

At $T=9 \mathrm{~K}$ the reflection $(0 \overline{2} 5)$ is split in both $\omega$ and $\theta$ while the reflection $(2 \overline{2} 0)$ remains sharp and (20 4) is split in $\omega$ only. These splittings imply a twinned monoclinic crystal with a monoclinic angle $\alpha$ different from $90^{\circ}$. Furthermore, they imply that the monoclinic distortion has occurred through a rotation of the $\mathbf{b}$ axis while the directions of the $\mathbf{a}$ and $\mathbf{c}$ axes are the same in both domains. 


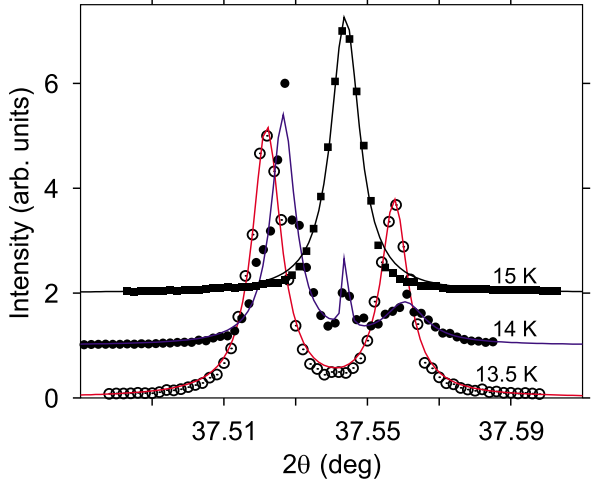

FIG. 7. (Color online) Diffracted intensity as a function of the scattering angle $2 \theta$ for the reflection $(0 \overline{2} 5)$ at selected temperatures. Lines represent fits to the data points with one, two, or three Lorentz-type functions at each temperature.

The experiment was continued by measuring $\omega-2 \theta$ maps of the $(0 \overline{2} 5)$ reflection by increasing the temperature in steps of $1 \mathrm{~K}$. The splitting remained visible up to $T=13.5 \mathrm{~K}$ and disappeared above $T=15 \mathrm{~K}$. At $14 \mathrm{~K}$ scattering centered at three different $2 \theta$ values was observed, which indicates the simultaneous presence of both the monoclinic lowtemperature and the orthorhombic high-temperature phases. These observations prove that the transition at $T_{N}$ is a firstorder phase transition. At $13.5 \mathrm{~K}$ both maxima appear broadened in the direction of $\omega$, suggesting that the transition might already have started. The transition temperature thus is estimated from the x-ray diffraction experiment as $T_{N}^{x}$ ray $=13.5 \pm 0.5 \mathrm{~K}$, in good agreement with the magnetization and heat-capacity results.

Diffracted intensity as a function of scattering angle was obtained by summing the measured intensities of the $\omega-2 \theta$ maps in the direction of $\omega$. By this we obtained pseudopowder-diffraction diagrams, which differ from real powder diffraction by the property that they do not contain diffraction by reflections other than $(0 \overline{2} 5)$ that might have similar scattering angles. Up to $T=13.5 \mathrm{~K}$ these plots contain two maxima that can be well fitted with two Lorentzian functions (Fig. 7). The difference in the center positions of the two Lorentzians provide an accurate estimate for the splitting in $2 \theta$ from which the monoclinic angle $\alpha$ can be calculated. ${ }^{19}$ The monoclinic distortion is weakly temperature dependent and corroborates the first-order character of the phase transition (Fig. 8).

At $9 \mathrm{~K} q$ scans were measured along $\mathbf{b}$ for six reflection pairs $(h k l) \rightarrow(h k+1 l)$. These indicated the presence of superlattice reflections at half-integer $k$ indices with the two highest intensities at $(0-0.53)$ and $(2-1.51) . \omega$ scans at these positions versus temperature showed the presence of superlattice reflections up to $13 \mathrm{~K}$ which disappeared above $15 \mathrm{~K}$ (Fig. 9).

\section{DISCUSSION}

Preceding work has indicated a phase transition to occur in $\mathrm{CrOCl}$ at low temperatures toward a state with AFM order on a fourfold magnetic supercell. ${ }^{8}$ Here we find that the AFM

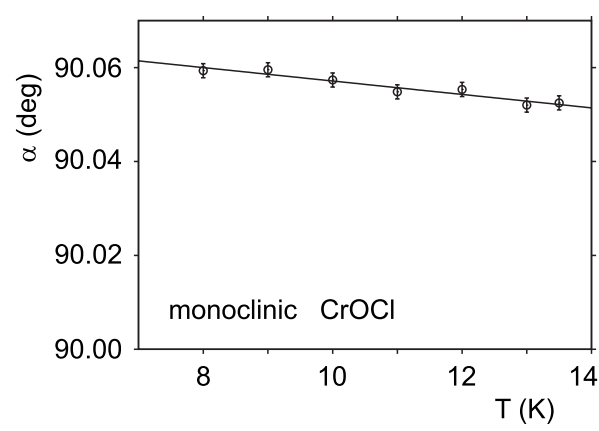

FIG. 8. Temperature dependence of the monoclinic angle $\alpha$ obtained from the splitting in $2 \theta$ of the reflection $(0 \overline{2} 5)$. The line represents a fit to the data points of $\alpha=\alpha_{0}+\alpha_{T} T$ with $\alpha_{0}$ $=90.071(22) \mathrm{deg}$ and $\alpha_{T}=-0.00143(18) \mathrm{deg} / \mathrm{K}$.

phase transition is accompanied by a lattice distortion toward monoclinic symmetry and by a structural distortion toward a twofold, $2 \mathbf{b}$, nuclear superstructure. The transition occurs at $T_{N}=13.5 \mathrm{~K}$, which is significantly lower than the magnetic transition at $\sim 80 \mathrm{~K}$ in $\mathrm{VOCl}$. The magnitude of the lattice distortion $\left(\alpha=90.06^{\circ}\right.$ for $\left.\mathrm{CrOCl}\right)$ is one third of that found in $\operatorname{VOCl}\left(\gamma=90.2^{\circ}\right.$ for VOCl $) .{ }^{14,15}$ Both features indicate a sizeable magnetoelastic coupling in $\mathrm{CrOCl}$ which is, however, considerably weaker than in $\mathrm{VOCl}$, despite the higher magnetic moment of $\mathrm{Cr}^{3+}$ compared to that of $\mathrm{V}^{3+}$. An explanation for the different behaviors of $\mathrm{CrOCl}\left(S=\frac{3}{2}\right)$, $\mathrm{VOCl}(S$ $=1)$, and TiOCl $\left(S=\frac{1}{2}\right)$ can be found in the different symmetries of the filled $3 d$ orbitals in these compounds.

The compounds $M \mathrm{OCl}(M=\mathrm{Ti}, \mathrm{V}, \mathrm{Cr})$ have a common topology and a common symmetry at the metal site. As a working hypothesis we therefore assume an equal hierarchy of $3 d$-orbital energies. For TiOCl, calculated electronic band structures have been reported at various levels of

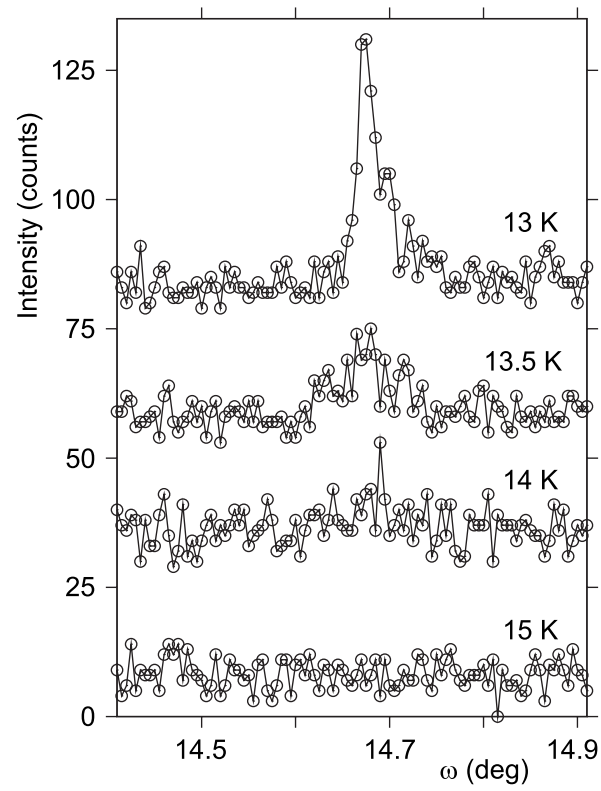

FIG. 9. $\omega$ scans centered at the superstructure reflection $(2-1.51)$ at selected temperatures. Given is the number of counts measured for $16 \mathrm{~s}$ in each step of $0.005^{\circ}$ wide. Scans at consecutive temperatures have been given an incremental off-set of 25 counts. 


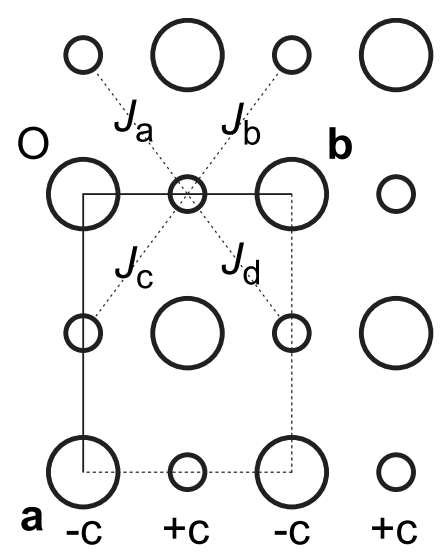

FIG. 10. Schematic representation of the $M O$ double layers projected onto the $a, b$ plane. Large circles denote oxygen atoms and small circles represent $M$ atoms. The unit cell of room-temperature structure is indicated. $+c$ and $-c$ denote atoms located at different coordinates along c. A monoclinic distortion with $\gamma \neq 90^{\circ}$ leads to exchange parameters $J_{a}=J_{d} \neq J_{b}=J_{c}$; a monoclinic distortion with $\alpha \neq 90^{\circ}$ leads to $J_{a}=J_{c} \neq J_{b}=J_{d}$.

theory. ${ }^{3,20,21}$ It has been found that the single valence electron of $\mathrm{Ti}^{3+}$ occupies the $3 d_{x^{2}-y^{2}}$ orbital-for $x$ along $\mathbf{b}, y$ along $\mathbf{c}$, and $z$ along $\mathbf{a}$ - that is responsible for AFM coupling by direct exchange between neighboring Ti atoms along $\mathbf{b}$. The orbitals next higher in energy are $3 d_{x z}$ and $3 d_{y z} \cdot 3,20,21$ These orbitals are empty in $\mathrm{TiOCl}$. Here we assume that the two valence electrons of $\mathrm{V}^{3+}$ in $\mathrm{VOCl}$ occupy the $3 d_{x^{2}-y^{2}}$ and $3 d_{x z}$ orbitals $^{15}$ and that the three valence electrons of $\mathrm{Cr}^{3+}$ in $\mathrm{CrOCl}$ occupy the $3 d_{x^{2}-y^{2}}, 3 d_{x z}$, and $3 d_{y z}$ orbitals. This arrangement explains AFM coupling along $\mathbf{b}$ and strong exchange interactions between neighboring chains of metal atoms through the electrons in the $3 d_{x z}$ and $3 d_{y z}$ orbitals.

$\mathrm{VOCl}$ has been found to develop an c-axis unique monoclinic lattice distortion. ${ }^{14,15}$ A closer inspection of the crystal structure suggests that this will be the most efficient lattice distortion in order to lift the degeneracy of exchange interactions on the $M \mathrm{O}$ bilayers involving the $3 d_{x z}$ orbitals of $\mathrm{V}$. This results in stripes of short $M-M$ contacts in the diagonal direction $\frac{1}{2} \mathbf{a}+\frac{1}{2} \mathbf{b}$ and stripes of long $M-M$ contacts in the direction $\frac{1}{2} \mathbf{a}-\frac{1}{2} \mathbf{b}$ (Fig. 10). AFM order along $\frac{1}{2} \mathbf{a}+\frac{1}{2} \mathbf{b}$ stripes then combines well with AFM order along $\mathbf{b}$ as imposed by direct exchange via the $3 d_{x^{2}-y^{2}}$ orbitals. The result is AFM order along a. The same distortion also lifts the degeneracy of exchange interactions involving $3 d_{y z}$ orbitals. However, AFM exchange interaction between these orbitals is probably enhanced along the direction $\frac{1}{2} \mathbf{a}-\frac{1}{2} \mathbf{b}$ of elongated bonds because $\sigma$-type overlap between $3 d_{y z}$ orbitals on neighboring metal atoms is increased in this direction. It thus appears that an c-axis unique monoclinic distortion leads to frustration between exchange interactions via the $3 d_{x z}$ orbitals (AFM interactions along $\frac{1}{2} \mathbf{a}+\frac{1}{2} \mathbf{b}$ ) and $3 d_{y z}$ orbitals (AFM interactions along $\frac{1}{2} \mathbf{a}-\frac{1}{2} \mathbf{b}$ ). As both orbitals are occupied in $\mathrm{CrOCl}$, the c-axis unique monoclinic distortion is unfavorable for this compound.

Experimentally, we have found an a-axis unique monoclinic lattice distortion for $\mathrm{CrOCl}$, in agreement with the above interpretation. Structurally the easiest way to realize a distortion of this kind requires a relative shift of neighboring layers $\mathrm{MOCl}$. However, this would hardly affect the frustrated exchange interactions within each layer. Instead, we have found that the monoclinic lattice distortion represents a shearing of individual layers, which then is responsible for the lifting of the degeneracy of exchange interactions on the $M O$ double layers. Rotation of $\mathbf{b}$ in the $(\mathbf{b}, \mathbf{c})$ plane results in shorter $M-M$ contacts that form a zigzag pattern on the average parallel to a. Direct exchange (AFM coupling) is increased along the shorter $M-M$ contacts for both the $3 d_{x z}$ and the $3 d_{y z}$ orbitals. The result is a ferromagnetic arrangement along a, which might explain the predominant FM exchange as derived from Curie-Weiss temperature. This order can be combined by AFM coupling between neighboring $M$ atoms along $\mathbf{b}$. The role of the small structural distortion (doubling of the $\mathbf{b}$ axis) could be to increase the exchange interactions between the zigzag stripes, however a more detailed description requires a complete structure model of the lowtemperature phase. It is noticed that nuclear superstructures with a wave vector that is twice the wave vector of the magnetic superstructure can be induced by the magnetic superstructure, as it has been explained theoretically. ${ }^{22}$ Incidentally, applying the same reasoning to the twofold magnetic superstructure of $\mathrm{VOCl}$, predicts the absence of a nuclear superstructure, in agreement with the experiment. ${ }^{15}$

The specific-heat data indicate additional transitions at a temperature of $\sim 27.2 \mathrm{~K}$ (Fig. 3). A signature of this magnetic transition has not been found in our $\mathrm{x}$-ray diffraction data while a weak anomaly is present in $\chi_{M}(T)$ (at the arrow in Fig. 2). This suggest a purely magnetic character of this transition, for example, the formation of incommensurate magnetic order.

While an c-axis monoclinic distortion appears to provide a maximum effect on lifting the degeneracy of the coupling between $3 d_{x z}$ orbitals of metal atoms on neighboring ribbons, it does not do so for the $3 d_{y z}$ orbitals. Instead, an a-axis monoclinic distortion through a change in direction of the $\mathbf{b}$ axis-i.e., representing a distortion of the layers rather than a relative shift between layers-accompanied by a structural distortion representing a doubling of the $\mathbf{b}$ axis leads to the required lifting of degeneracy of exchange interactions through the $3 d_{y z}$ orbitals. The different lattice distortions of $\mathrm{VOCl}$ and $\mathrm{CrOCl}$ are thus explained by the different symmetries of the filled $3 d$ orbitals.

\section{CONCLUSIONS}

The phase transition of $\mathrm{CrOCl}$ toward a state of AFM order has been identified as a first-order phase transition at $T_{N}=13.5 \mathrm{~K}$, which is accompanied by a lattice and a structural distortion toward a twofold, $2 \mathbf{b}$ superstructure with aaxis unique monoclinic symmetry. The ordered magnetic moment is parallel to c. A second transition is discovered that is presumably of purely magnetic origin and might indicate the formation of an incommensurate magnetic superstructure.

The different behaviors of $\mathrm{TiOCl}, \mathrm{VOCl}$, and $\mathrm{CrOCl}$ result from different symmetries of the occupied $3 d$ orbitals, which lead to different exchange interactions on the $M O$ double layers of these isostructural series of compounds. 


\section{ACKNOWLEDGMENTS}

Single crystals of $\mathrm{CrOCl}$ have been grown by A. Suttner. We gratefully acknowledge W. Morgenroth for assistance with the x-ray diffraction experiments with synchrotron ra- diation at Hasylab and E. Brücher and G. Siegle with magnetization and heat-capacity measurements. Financial support has been provided by the German Science Foundation (DFG). *smash@uni-bayreuth.de; www.crystal.uni-bayreuth.de

${ }^{1}$ A. Seidel, C. A. Marianetti, F. C. Chou, G. Ceder, and P. A. Lee, Phys. Rev. B 67, 020405(R) (2003).

${ }^{2}$ M. Shaz, S. van Smaalen, L. Palatinus, M. Hoinkis, M. Klemm, S. Horn, and R. Claessen, Phys. Rev. B 71, 100405(R) (2005).

${ }^{3}$ T. Saha-Dasgupta, R. Valenti, H. Rosner, and C. Gros, Europhys. Lett. 67, 63 (2004).

${ }^{4}$ H. Schäfer and F. Wartenpfuhl, Z. Anorg. Allg. Chem. 308, 282 (1961).

${ }^{5}$ P. Ehrlich and H.-J. Seifert, Z. Anorg. Allg. Chem. 301, 282 (1959).

${ }^{6}$ A. Haase and G. Brauer, Acta Crystallogr., Sect. B: Struct. Crystallogr. Cryst. Chem. 31, 2521 (1975).

${ }^{7}$ M. D. Lind, Acta Crystallogr., Sect. B: Struct. Crystallogr. Cryst. Chem. 26, 1058 (1970).

${ }^{8}$ A. N. Christensen, T. Johansson, and S. Quézel, Acta Chem. Scand. Ser. A 28, 1171 (1975).

${ }^{9}$ A. Adam and G. Buisson, Phys. Status Solidi A 30, 323 (1975).

${ }^{10}$ A. Wiedenmann, J. P. Venien, P. Palvadeau, and J. RossatMignod, J. Phys. C 16, 5339 (1983).

${ }^{11}$ S. van Smaalen, L. Palatinus, and A. Schonleber, Phys. Rev. B 72, 020105(R) (2005).

${ }^{12}$ A. Krimmel, J. Strempfer, B. Bohnenbuck, B. Keimer, M. Hoinkis, M. Klemm, S. Horn, A. Loidl, M. Sing, R. Claessen, and M. v. Zimmermann, Phys. Rev. B 73, 172413 (2006).

${ }^{13}$ A. Schönleber, S. van Smaalen, and L. Palatinus, Phys. Rev. B 73, 214410 (2006).

${ }^{14}$ A. C. Komarek, T. Taetz, M. T. Fernandez-Diaz, D. M. Trots, A. Möller, and M. Braden, Phys. Rev. B 79, 104425 (2009).

${ }^{15}$ A. Schönleber, J. Angelkort, S. van Smaalen, L. Palatinus, A. Senyshyn, and W. Morgenroth, Phys. Rev. B 80, 064426 (2009).

${ }^{16}$ K. Nocker and R. Gruehn, Z. Anorg. Allg. Chem. 619, 699 (1993).

${ }^{17}$ P. W. Selwood, Magnetochemistry, 2nd ed. (Interscience, New York, 1956).

${ }^{18}$ A. Abragam and B. Bleaney, Electron Paramagnetic Resonance of Transition Ions (Oxford University Press, Oxford, 1970).

${ }^{19}$ A. Schönleber, G. Shcheka, and S. van Smaalen, Phys. Rev. B 77, 094117 (2008).

${ }^{20}$ D. Fausti, T. T. A. Lummen, C. Angelescu, R. Macovez, J. Luzon, R. Broer, P. Rudolf, P. H. M. van Loosdrecht, N. Tristan, B. Buchner, S. van Smaalen, A. Möller, G. Meyer, and T. Taetz, Phys. Rev. B 75, 245114 (2007).

${ }^{21}$ Y.-Z. Zhang, H. O. Jeschke, and R. Valenti, Phys. Rev. Lett. 101, 136406 (2008).

${ }^{22}$ S. W. Lovesey and S. P. Collins, X-ray Scattering and Absorption by Magnetic Materials (Clarendon, Oxford, 1996). 